\title{
Research on Strategies of Low-Carbon City Planning and Construction
}

\author{
Min Zhang $^{1}$ \\ ${ }^{1}$ Tianjin University of Technology, Tianjin, China
}

\begin{abstract}
With the continuous development of China's economy, the policy of sustainable development has increasingly become the leading direction of urban planning and construction at all levels, and the concept of low-carbon city construction continues to emerge. In the process of low-carbon city construction, it is required to start from reducing carbon emissions, uphold the five concepts of innovation, coordination, greenness, openness, and sharing, explore low-carbon eco-city planning strategies and methods, and make urban planning become the local government's emission reduction measures an important means to make the city's low-carbon development more smoothly and realize the sustainable development of low-carbon cities.
\end{abstract}

\section{Introduction}

Cities are the most important centers of economic and social activities in China, as well as an important source of greenhouse gases. and they are also the most sensitive places affected by climate change. With the deepening and accelerating of China's urbanization process, the increase of urban population will inevitably lead to the growth of energy consumption. Therefore, urban development of low-carbon economy is an important measure to solve the problems of climate change and sustainable energy development in China. Low-carbon city is an urban development concept of low-carbon consumption and production in cities, which can help establish a more economical and friendly urban energy ecological system. Low-carbon cities not only require cities to pay attention to economic development, but also to control greenhouse gas emissions and energy consumption at a low level. The traditional energy methods should be reformed and innovated to reduce the consumption of high-carbon energy and the emission of urban greenhouse gases, so as to achieve a win-win situation of urbanization development and ecological environment protection ${ }^{[1]}$. The construction of low-carbon city has become one of the most important development directions recognized by all countries at present, and the future development of lowcarbon city will also become the focus of global attention.

\section{Evaluation criteria for low-carbon cities}

The evaluation of the level of urban low-carbon development can use multiple indicators such as the level of economic development, the living standards of residents, and the intensity of carbon emissions. Comparatively speaking, it is more reasonable to use the ratio coefficient between annual per capita GDP growth rate and $\mathrm{CO}_{2}$ emission growth rate, namely the elasticity coefficient, to evaluate the effectiveness of low-carbon city construction. Based on the elasticity coefficient, the development level of low-carbon cities can be divided into three levels: no decoupling, relative decoupling and absolute decoupling ${ }^{[2]}$ :

1) No decoupling. If the elasticity coefficient is greater than or equal to 1 , the growth rate of carbon emission is equal to or greater than the growth rate of economic growth.

2) Relative decoupling. If the elasticity coefficient is less than or equal to 0.50 , the growth rate of carbon emission is less than half of the economic growth rate.

3) Absolute decoupling. If the elasticity coefficient is less than or equal to 0 , the annual per capita GDP keeps growing, while the $\mathrm{CO}_{2}$ emissions remain unchanged or show negative growth.

Regarding the calculation of $\mathrm{CO}_{2}$ emissions, the accounting method provided by IPCC can be adopted according to the usage of different types of energy: $\mathrm{CO}_{2}=K^{*} E$

In the formula, $E$ is the consumption of different types of energy converted from standard coal, and $K$ is the carbon emission coefficient.

\section{Key points of low-carbon city construction}

\subsection{Develop low-carbon energy}

3.1.1 Improve resource utilization and promote the transformation of energy from high-carbon to lowcarbon

In the process of economic development, China is not only 
highly dependent on natural resources, but also facing the situation of lack of natural resources. China's energy structure is dominated by coal, which has occupied the position of the main energy consumption for a long time. Therefore, it is necessary to conduct low-carbon energy treatment in order to achieve the purpose of controlling greenhouse gas emissions. To further control the development of the technology of producing hydrogen from coal by using the idea of increasing income and reducing expenditure, so that coal can be used efficiently and cleanly, the pressure of carbon emission can be further reduced ${ }^{[3]}$.

\subsubsection{Change the energy structure and vigorously develop renewable energy}

At present, carbon containing fossil energy is the main body of energy consumption in China such as oil, natural gas, coal, and etc. ${ }^{[4]}$. The construction of low-carbon city needs to change the energy structure and vigorously develop renewable energy. Renewable energy includes hydro energy, biomass energy, wind energy, nuclear energy, solar energy, tidal energy, geothermal energy and ocean energy, etc., which have great potential. It is necessary to further develop the acquisition and application of renewable energy, increase the proportion of sustainable renewable energy in China's energy structure, and effectively relieve the pressure of carbon emissions. Second, the appropriate development of nuclear power. Nuclear power, hydropower and thermal power are known as the world's three pillars of power generation energy. On the premise of ensuring the safety of nuclear technology, nuclear power plants should be rationally distributed and the development of nuclear power should be accelerated.

\subsection{Design green transportation planning}

\subsubsection{Advocate green travel mode to reduce environmental pollution}

As shown in Table 1, there is a large gap in energy consumption and waste emissions among different modes of transportation. Incorporating the concept of green transportation into urban road traffic, we should vigorously guide the green transportation system based on "bus + walking". These modes of travel have the advantages of small footprint, low energy consumption, and low pollution, and emphasis on "green" of the urban traffic. The number of private cars should be strictly controlled and the rate of car trips should be controlled within a reasonable range. For example, the operation system of odd-even license plates should be implemented. The transfer space of buses and bicycles in the subway and main roads should be optimized so as to reduce traffic energy consumption and air pollution. Through the establishment and maintenance of urban sustainable development of the traffic system, to meet the traffic needs of people, with the least social cost to achieve the maximum traffic efficiency.
Table 1. Energy consumption and exhaust emissions of different modes of transportation.

\begin{tabular}{|c|c|c|}
\hline $\begin{array}{c}\text { Traffic } \\
\text { tools }\end{array}$ & $\begin{array}{c}\text { Emissions } \\
\left(\mathbf{g} \cdot \mathbf{k m}^{-\mathbf{1}}\right)\end{array}$ & $\begin{array}{c}\text { Energy consumption } \\
\mathbf{( k w} \cdot \mathbf{h} \cdot \mathbf{k m}^{-1} \mathbf{)}\end{array}$ \\
\hline Car & 18.9 & 0.28 \\
\hline Motorcycle & 27.4 & 0.55 \\
\hline Bus & 0.9 & 0.09 \\
\hline Light rail & 0.6 & 0.05 \\
\hline Bicycle & 0 & 0.06 \\
\hline Walking & 0 & 0.04 \\
\hline
\end{tabular}

\subsubsection{Optimize the land use around the site}

As shown in Table 2, TOD nodes are constructed by rail transit and BRT stations, which are taken as the core to form a beaded development axis, and drive the development of surrounding land to form a development belt. Taking the opportunity of the construction of largevolume BRT, layout and density of the land along the route should be adjusted accordingly to optimize the urban land use. To build a modern urban space with residents' living and travel modes closely integrated, so as to increase the number of residents and employed people in the core area of the station, and increase the passenger flow of urban mass bus rapid transit lines. Reasonably develop transportation, and establish a differentiated development model in accordance with the radiation range of rail transit and BRT.

Table 2. TOD development mode of core area

\begin{tabular}{|c|l|}
\hline Region & \multicolumn{1}{c|}{ Development mode } \\
\hline $\begin{array}{c}\text { High strength } \\
\text { development zone }\end{array}$ & $\begin{array}{l}\text { The 500m radius around the railway } \\
\text { station is taken as the key influence } \\
\text { area, ultra-high intensity development } \\
\text { can be carried out within 300m radius, } \\
\text { and high-intensity development can be } \\
\text { carried out between 300m and 500m. }\end{array}$ \\
\hline $\begin{array}{c}\text { Medium and high } \\
\text { strength }\end{array}$ & $\begin{array}{l}\text { The 1000m area along the track and } \\
\text { 500m area along the BRT are the } \\
\text { secondary impact areas, and the } \\
\text { secondary high-intensity development } \\
\text { is to build a series of bus traffic } \\
\text { communities with the track stations or } \\
\text { BRT stations as the core. }\end{array}$ \\
\hline $\begin{array}{c}\text { Middle and low } \\
\text { strength }\end{array}$ & $\begin{array}{l}\text { In the railway station outside the scope } \\
\text { of 1000m and BRT500m outside the } \\
\text { area, basically large transport capacity } \\
\text { bus cannot directly radiation range, } \\
\text { conventional bus as the main traffic } \\
\text { service mode, adopt low and medium } \\
\text { intensity development. }\end{array}$ \\
\hline
\end{tabular}

\subsection{Measures to increase carbon sink}

Urban "carbon sink" system mainly comes from water area, woodland, grassland, garden and other ecological land. As shown in Fig. 1, factors such as the area of 
ecological land and the rate of green land are mainly considered in the control planning, while the control and planning indexes of green urban area are mainly for all kinds of green land in the city and the area of large trees in the construction land. Taking into account these " regulation " elements, the following measures are proposed to reduce carbon emissions:

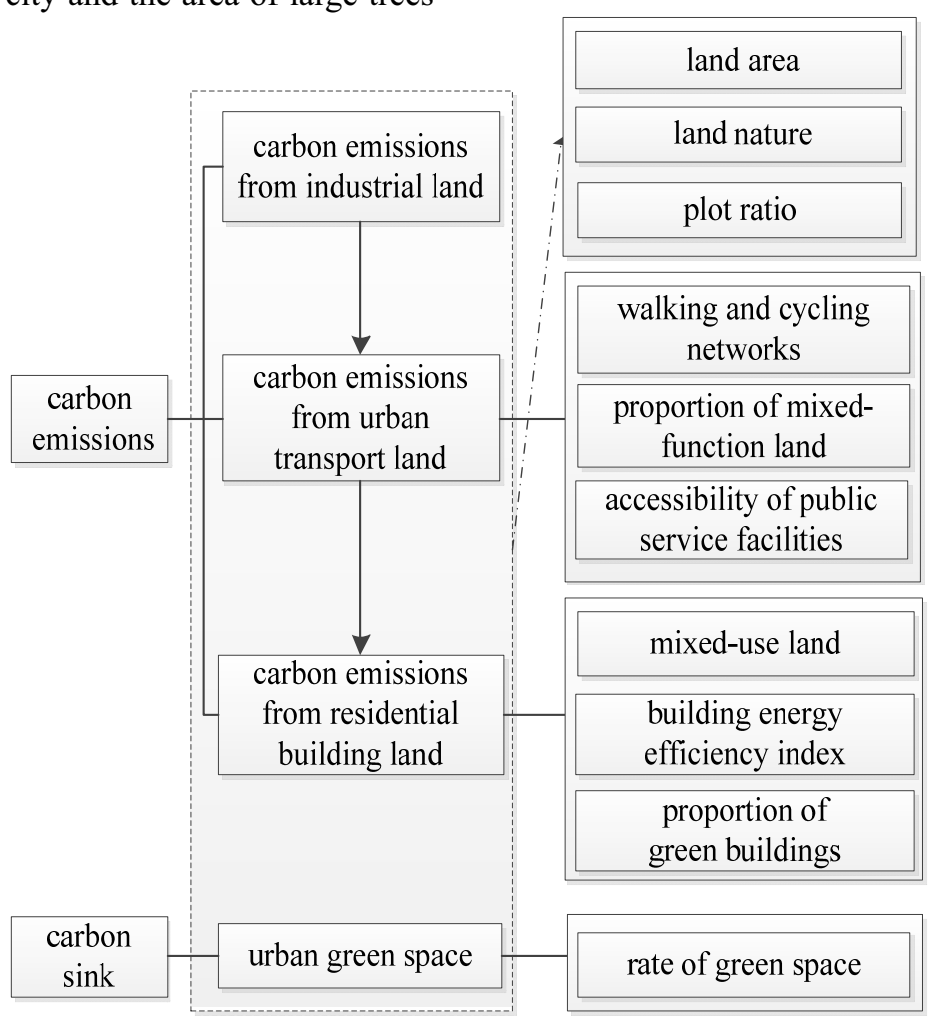

Fig. 1. Analysis of elements related to regulation in carbon balance.

\subsubsection{Improve the area of green space in urban centers}

According to the types of land used in the planning, the area of public green space, protective green space, park green space, square green space, road green space and garden green space for production shall be increased, and the rate of unit green land shall also be increased. In order to ensure a good ventilation environment in the core area of the urban area, the building density should be reduced in the area shaded by the dominant wind direction, the coverage rate of green space and the permeable ground area should be increased. At the same time, the building form and layout should be controlled in the urban design to avoid the formation of roadway with excessive local wind speed.

\subsubsection{Reasonably plan the layout of construction land}

In order to improve the carbon sink capacity per unit green area, it is beneficial to increase the coverage area of arbor in construction land, promote the combination of arbor, forest and wood, and construct the three-dimensional plant community structure dominated by arbor in construction land. Attention should be paid to controlling noise sources and reasonable layout of noise-sensitive buildings in residential land near the central business district and mixed land including residential land. Isolation or noise reduction measures, such as low-noise road surface, road sound barrier, greening and noise reduction, and restriction of heavy-duty vehicles, should be adopted in areas with large traffic noise impact to reduce environmental noise interference.

\subsubsection{Emphasize three-dimensional greening in urban green buildings}

In urban buildings, it is advisable to afforestation the living room and the roof to improve the ecological benefits of the city. Increase the forest area around the city, and build a green ecological barrier. The design and construction of buildings must be constructed in accordance with building energy-saving standards, and a timely energy monitoring system must be configured. In terms of building energy efficiency design, the solar roof program must be promoted vigorously. Nowadays, the price of solar panels and raw materials has fallen a lot. At the same time, this situation can be used as an opportunity to increase the use of solar energy and promote the use of solar photovoltaic buildings. Through the sponge city concept, urban construction is introduced to make reasonable use of water resources and ensure water circulation ${ }^{[5]}$

\section{Conclusion}

Low-carbon city planning and construction should start from the current status of the city, improve the working 
system of low-carbon city construction, innovate the concept of low-carbon city construction, and truly integrate the low-carbon concept into the details of urban development and construction. At present, China's lowcarbon city construction started late, and there is still a gap with the world's developed countries. On the one hand, we should face up to this gap and carry out the construction and development of low-carbon cities according to actual conditions. On the other hand, we should strengthen the study of international excellent environmental protection concepts and technologies, accelerate the construction and development of low-carbon cities in China, and further promote the harmonious development of cities and natural environment.

\section{References}

1. Yan Gao. Housing and Real Estate. 58 (2018)

2. Fei Chen, Dajian Chu. Urban Development Studies. 16, 71-79 (2009)

3. Baojuan Ma. Low Carbon World. 31-32(2018)

4. Hailiang Ma, Gelin Zhang. Soft Science. 33,101104+117(2019)

5. Xu Sun, Xiao Han. Housing and Real Estate. 166(2017) 